\title{
Critical cluster size for mixed valence in small matrix-isolated Sm clusters
}

\author{
W. Niemann, ${ }^{*}$ W. Malzfeldt ${ }^{\dagger}$ P. Rabe, ${ }^{\ddagger}$ and R. Haensel ${ }^{\S}$ \\ Institut für Experimentalphysik, Universität Kiel, D-2300 Kiel, Federal Republic of Germany \\ M. Lübcke** \\ II. Institut für Experimentalphysik, Universität Hamburg, D-2000 Hamburg 50, Federal Republic of Germany
}

(Received 6 May 1986)

\begin{abstract}
From $L_{3}$ absorption measurement, information on the valence and size of small matrix-isolated Sm clusters is obtained by analyzing the x-ray-absorption near-edge structure and the extended $x$ ray-absorption fine structure (EXAFS). Depending on the mean cluster size, which is a function of the atomic concentration ( $\mathrm{Sm}$ to rare-gas atom ratio), every valence between 2.0 and 3.0 is reproducibly achievable. At very low Sm concentrations the samples have the atomic valence; at a critical concentration $c \approx 1: 100$ a steep rise of the valence up to $v=2.6$ is found. Then, with increasing metal concentration, the Sm-cluster valence approaches the valence of the solid. From EXAFS analysis, a mean cluster size of 13 atoms for clusters with $v=2.6$ follows. The mixed valence of the $\mathrm{Sm}$ atoms is explained by the pressure exerted on the $\mathrm{Sm}$ cluster by the distorted rare-gas lattice (the rare gas is $\mathrm{Ne}, \mathrm{Ar}$, or $\mathrm{Kr}$ ).
\end{abstract}

\section{INTRODUCTION}

The phenomenon of a mixed valence in materials containing elements of the rare-earth group has been the subject of numerous investigations in the recent past. $^{1-3}$ Possible methods allowing valence changes from the divalent $4 f^{N}\left(5 d^{0} 6 s^{2}\right)$ configuration to the trivalent $4 f^{N-1}\left(5 d^{1} 6 s^{2}\right)$ configuration in a given sample are highpressure experiments or temperature changes. Additionally, valence changes are achievable by chemical substitution of a fraction of the sample material or by varying the local coordination around the rare-earth atoms. From photoemission experiments, coordination-dependent valences have been clearly demonstrated for bulk Sm material $^{4-6}$ for Sm clusters evaporated onto different substrates, ${ }^{7,8}$ and for Tm metal. ${ }^{9}$

In this paper, a new method for the investigation of coordination-dependent valences is used. By means of the matrix isolation technique, small $\mathrm{Sm}$ clusters isolated in matrices of solid neon, argon, or krypton are prepared. By strongly varying the metal concentration, the whole transition (isolated atom to small cluster to big cluster to bulk material) can be studied. The existence of a divalent $\mathrm{Sm}$ surface on a trivalent $\mathrm{Sm}$ bulk material ${ }^{10}$ renders $\mathrm{Sm}$ clusters especially interesting.

A drastic valence change of small Sm clusters isolated in solid argon has already been observed. ${ }^{11}$ In this paper the influence of different matrices on the valence and the determination of the Sm-cluster sizes from the analysis of the extended $x$-ray-absorption fine-structure (EXAFS) data are presented. A further study on $\mathrm{Nd}$ and $\mathrm{Pr}$ clusters isolated in matrices of solid argon is published elsewhere. $^{12}$

In order to study the cluster size dependence of the electronic configuration of matrix-isolated $\mathrm{Sm}$ clusters, $L_{3}$ absorption spectra have been taken. The mean valence of the clusters is determined by analyzing the near-edge structures (white lines) at the $L_{3}$ edge, whereas information on the mean cluster size is obtained from the corresponding EXAFS data.

In Sec. II, the experimental setup used for the absorption measurements is described. Detailed information on the sample chamber and on the conditions for the sample preparation is given. Experimental results are presented in Sec. III, together with the methods used for the determination of the valences from the $L_{3}$ x-ray-absorption near-edge structure (XANES) spectra and for the determination of the mean cluster size from the EXAFS data. In Sec. IV the size-dependent valence of small matrixisolated Sm clusters is discussed.

\section{EXPERIMENTAL SETUP}

The experiments have been performed at the EXAFS II beamline of the Hamburger Synchrotronstrahlungslabor (HASYLAB). Detailed information on this beamline has already been published elsewhere ${ }^{13-15}$ so that we will restrict ourselves to a short description of the general beamline but present detailed information on the sample chamber and the sample preparation.

In a windowless UHV beamline $\left(p \sim 10^{-10} \mathrm{hPa}\right)$ the synchrotron radiation is focused onto the sample in a 1:1 image by a unit of four toroidal mirrors. The arrangement of the optical elements has been chosen in such a way that first the polychromatic radiation is reflected by the mirror unit onto the monochromator which has a distance of $36 \mathrm{~m}$ to the storage ring, and then the monochromatic light enters the sample chamber and the detectors. The surface of the mirror elements is coated by a thin $\mathrm{Au}$ film resulting in a critical energy $E_{c}=12 \mathrm{keV}$ for a glancing angle $\theta=7 \mathrm{mrad}$. A sufficient photon flux is achieved for energies up to $15 \mathrm{keV}$.

The double-crystal monochromator of the JUMBOtype $^{16}$ is equipped with two $\mathrm{Si}(111)$ crystals which are 
moved independently. A constant exit beam is achieved by adding a translational movement of the second crystal to the obligatory two rotations. In the vicinity of the $\mathrm{Sm}$ $L_{3}$ edge $(E \sim 6.7 \mathrm{keV})$, the energy resolution is adjustable to $\Delta E \sim 1 \mathrm{eV}$ by carefully closing the slits in front of the monochromator. The focusing beamline allows a flux of up to $10^{11}$ photons $/(\sec \Delta E)$ at the sample within a band pass of $3 \mathrm{eV}$.

The detection system consists of three ionization chambers mounted in a row with two sample chambers placed between them, enabling the simultaneous measurement of the absorption spectra of two different samples. In the first sample chamber the matrix is prepared, whereas in the second one a reference sample which has been chosen to be $\mathrm{Sm}_{2} \mathrm{O}_{3}$ is installed. By simultaneously measuring the absorption of the matrix and of the reference, the energy scale of the matrix spectrum is calibrated relative to the position of the edge of the reference which has been defined to be $6.716 \mathrm{keV}$. This method compensates for variations of the actual energy of two successive matrix spectra due to small changes of the vertical position of the synchrotron beam source. The ionization chambers are filled with nitrogen, resulting in an absorption of $10-15 \%$ in each of them. The setting of the monochromator and the data accumulation are controlled by a Digital Equipment Corporation PDP11/23 computer via a CAMAC (computer-aided measurement and control) interface. $^{15}$

\section{Sample chamber and sample preparation}

The samples have been prepared in a UHV chamber, shown in Fig. 1, which is pumped by a 100 liter/sec turbomolecular pump. At room temperature a residual pressure of $p=10^{-8} \mathrm{hPa}$ is achieved. Thin Be windows (50 $\mu \mathrm{m}$ ) protect the sample chamber from the gases of the two neighboring ionization chambers. A $\mathrm{He}$ cryostat mounted on top of the sample chamber is equipped with a special sample holder to take up a thin aluminum foil on which the metal and the matrix gas are frozen. At the normal operational temperature, $5 \mathrm{~K}$, the residual pressure in the sample chamber drops down to $10^{-9} \mathrm{hPa}$. The sample holder is surrounded by a cooling shield $(T \sim 40$ $\mathrm{K})$ which has several openings to pass the $\mathrm{X}$-ray beam horizontally and to prepare the sample by vertical atomic beams. A mass spectrometer analyzes the composition of the residual gas in the chamber and the purity of the matrix gas. In the bottom of the sample chamber the watercooled atomic beam source for the evaporation of the metal atoms is attached. A system of slits above the beam source prevents the cryostat from getting a large heat load. The shutter below the sample holder is only opened during the evaporation. The input of the matrix gas is enabled by a high-precision valve followed by a thin tube ending approximately $100 \mathrm{~mm}$ below the sample holder. For the evaporation of Sm, which needs a temperature of $\sim 1000 \mathrm{~K}$ (Ref. 17) in order to get a vapor pressure of $10^{-2} \mathrm{hPa}$, a resistively heated furnace is used. The distance from the sample holder to the furnace is $350 \mathrm{~mm}$. High purity gases (Messer-Griesheim, >99.999\%) and metal pieces (Universal Mathey, > 99.9\%) have been used for the preparation of the samples.

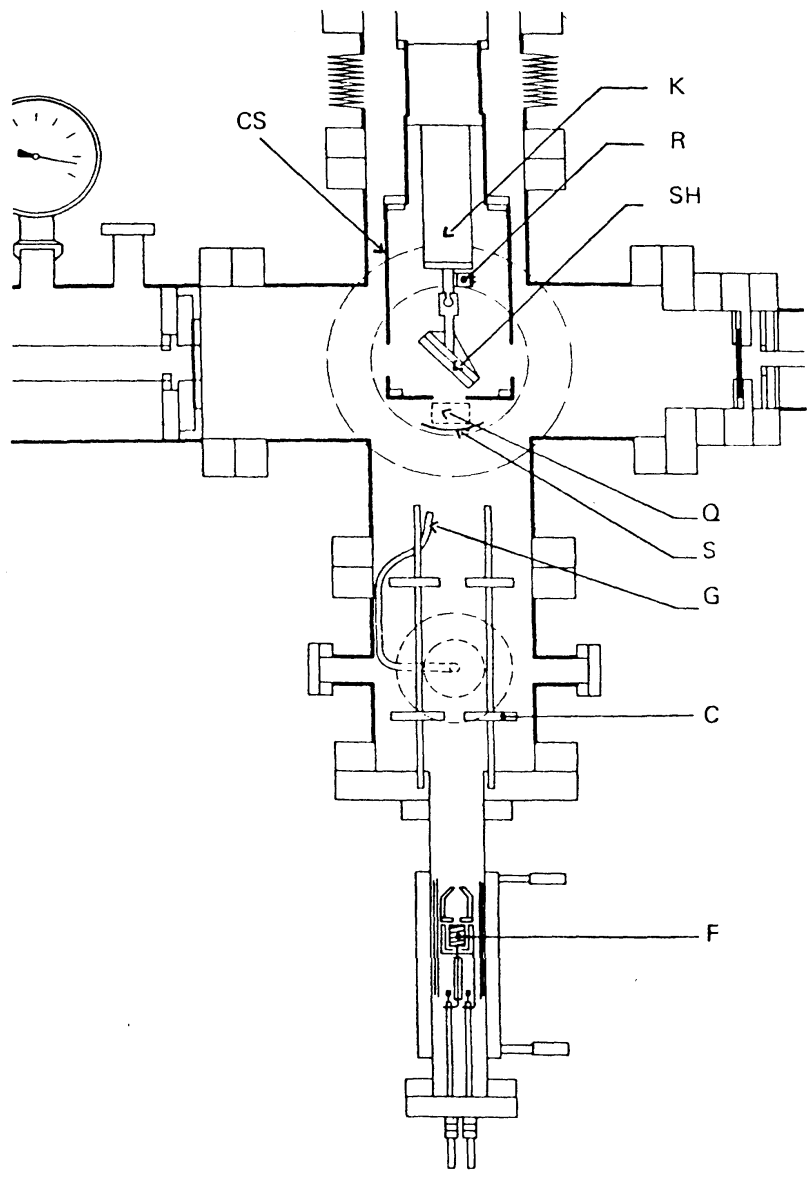

FIG. 1. Side view (vertical cut) of the sample chamber used for the matrix experiments. Parts of the equipment are explained by the following abbreviations: $\mathrm{K}$ : cryostat; CS: cooling shield; $\mathrm{R}$ : carbon resistor; $\mathrm{SH}$ : sample holder; $\mathrm{Q}$ : quartz; $\mathrm{S}$ : shutter; $G$ : tube for gas inlet; $C$ : fixed collimation; F: furnace.

Before preparing the samples, a careful outgasing of the metal pieces in the furnace has to be performed in order to eliminate impurities. After several hours of heating the sample, only a small fraction of $\mathrm{H}_{2}$ can be detected with the mass spectrometer. The amount of impurities $\left(\mathrm{H}_{2}\right)$ in the matrix is estimated to be less than $0.1 \%$ of the raregas material.

The matrices are prepared in situ by use of a sandwich technique. First a thin layer of rare gas $(\sim 100 \mathrm{~nm})$ is frozen onto the aluminum foil in order to prevent the metal atoms from reaching the surface of the foil. Then the metal atoms together with the rare gas (argon, neon, or krypton) are cocondensed. Afterwards a protective thin layer of rare gas is frozen onto the matrix. The ratio $M / R$ (matrix to radical composition ratio) of the number of atoms in the sample is used for characterization. $M / R$ is the reciprocal of the metal concentration. We will use this notation because it is commonly used in matrix isolation spectroscopy. Typical preparation parameters are a $\mathrm{Sm}$ deposition rate equal to $1-2 \mathrm{~nm} / \mathrm{min}$ and a rare-gas deposition rate equal to $50-400 \mathrm{~nm} / \mathrm{min}$. The thickness $d_{T}$ of the Al foil has been $5 \mu \mathrm{m}$; for some Ne matrices $d_{T}$ 
has been $25 \mu \mathrm{m}$. During the preparation procedure the metal deposition rate $\Delta D / \Delta t$ is monitored by a quartz crystal, and the gas deposition rate is measured indirectly via the pressure $\mathbf{p}_{\text {gas }}$ in the sample chamber, which during preparation is some orders of magnitude higher than the base pressure [typical values $\mathbf{p}_{\mathrm{gas}}=(2-150) \times 10^{-6} \mathrm{hPa}$. The gas pressure is adjusted such that the ratio $x=\mathbf{p}_{\text {gas }} /(\Delta D / \Delta t)$ is kept constant. There is a linear relationship $M / R=$ const $\times x^{1}$ which very easily allows one to obtain the desired $M / R$ as a function of the preparation parameters. During preparation the temperature in the matrix is less than $8 \mathrm{~K}$, since even the preparation of doped $\mathrm{Ne}$ matrices is possible. The temperature of the sample holder is measured by a $C$ resistor.

The thickness of the metallic portion of the sample is achieved by comparing the measured jump of the $\operatorname{Sm~} L_{3}$ edge with known cross sections, ${ }^{18}$ and after subtracting the absorption of the metal from the absorption spectrum before the edge, the thickness of the rare-gas matrix is determined similarly.

Within the typical preparation time of $2-6 \mathrm{~h}$, a Sm thickness of up to $500 \mathrm{~nm}$ has been obtained. The typical jump of $\Delta \mu d=0.05$ in the $\operatorname{Sm~} L_{3}$ edge is sufficient to measure good XANES spectra but poses extreme difficulties on the measurements of EXAFS spectra. The ratio $M / R$ has been varied from $M / R=6: 1$ to $M / R=450: 1$.

\section{DATA ANALYSIS AND EXPERIMENTAL RESULTS}

\section{Valence determination from $L_{3}$ XANES}

The absorption of the matrix samples is determined by measuring the intensities $I_{0}\left(I_{1}\right)$ of the x-ray beam traversing the first (second) ionization chamber in arrangement with and without sample:

$$
(\mu d)_{\text {sample }}=\ln \left[\frac{\left(I_{0} / I_{1}\right)_{\text {with sample }}}{\left(I_{0} / I_{1}\right)_{\text {without sample }}}\right] .
$$

The absorption spectrum without sample is taken immediately before starting the preparation of the matrix. The contribution due to the $\mathrm{Sm}$ absorption at the $L_{3}$ edge is obtained by subtracting the background which is due to higher-shell excitations of Sm and to absorption by the matrix from the data. This background is fitted by the well-known Victoreen formula. ${ }^{19}$

The $L_{3}$ absorption of matrix-isolated Sm clusters at $T=5 \mathrm{~K}$ is shown for the extreme cases of small matrix atoms (neon, $r=0.158 \mathrm{~nm}$ ) and large matrix atoms (krypton, $r=0.199 \mathrm{~nm}$ ) in Figs. 2 and 3. The data of Sm in argon have already been published. ${ }^{11}$

The prominent features in the near-edge region ("white lines") are attributed to transitions from the $2 p_{3 / 2}$ core level to unoccupied $5 d$ levels. The energy separation $\Delta E=7.5 \mathrm{eV}$ between the divalent and the trivalent white lines of $\mathrm{Sm}$ has first been observed in $\mathrm{SmB}_{6}{ }^{20}$

A comparison of the divalent matrix spectrum $(M / R=135)$ in Fig. 3 with the absorption spectrum of atomic Sm (Ref. 21) shows no differences. From an inspection of Figs. 2 and 3 it is clearly demonstrated that the whole range from divalent to nearly trivalent $\mathrm{Sm}$ clus-

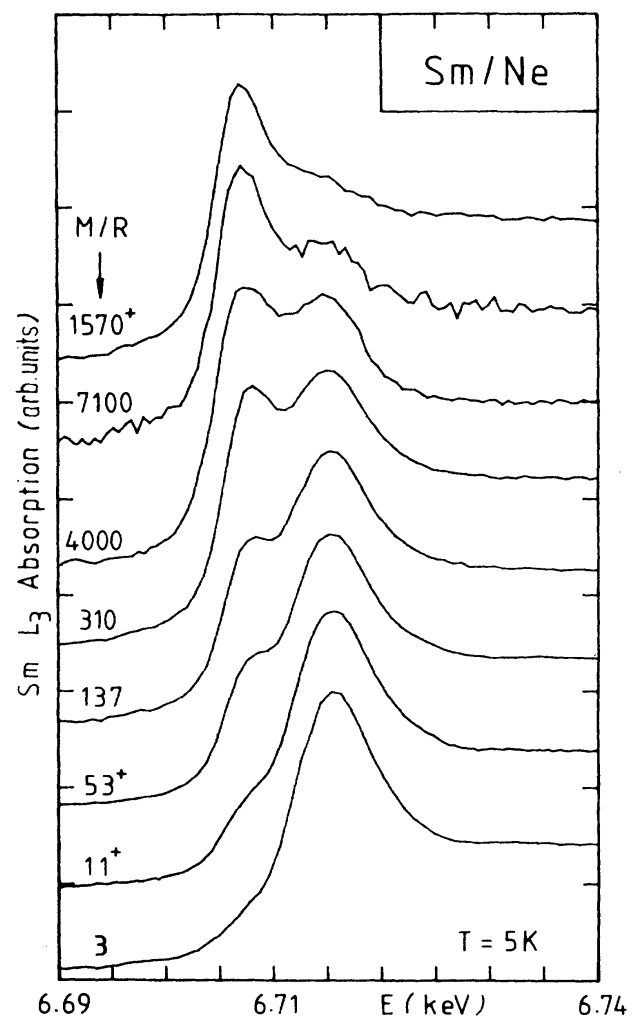

FIG. 2. $L_{3}$ absorption spectra of Sm clusters in neon matrices at $T=5 \mathrm{~K}$. The concentration $M / R$ (matrix to radical) varies from 7100:1 to 3:1. The thickness of the Al support was $d_{T}=5 \mu \mathrm{m}$, in some cases $(+) d_{T}=25 \mu \mathrm{m}$.

ters can be investigated.

The average valence of the Sm clusters in a given sample can be extracted by fitting a superposition of divalent and trivalent $\mathrm{Sm}$ spectra to the measured data. This method has been used successfully for the analysis of the $\mathrm{Sm} / \mathrm{Ar}$ data. ${ }^{11}$ However, in this publication a different (more flexible) method yielding the same results for the determination of the valence is used. The white lines at the $L_{3}$ threshold are fitted by analytical Voigt profiles, ${ }^{22,15}$ whereas contributions of transitions to continuum states are accounted for by arctangent functions. The method of fitting the near-edge structures by discrete functions generally has been used successfully to determine the valence ${ }^{23}$ of mixed-valent systems. In this case, the valence is defined by

$$
V=2+H^{\text {triv }} /\left(H^{\text {div }}+H^{\text {triv }}\right),
$$

where $H^{\text {div }}\left(H^{\text {triv }}\right)$ is the arctangent amplitude of divalent (trivalent) $\mathrm{Sm}$. In order to get reliable fit parameters it is necessary to keep the ratio $H$ (Voigt) $/ H\left(\tan ^{-1}\right)$ constant for every valence state.

The results of the fit procedure used to determine the mean valence of the Sm clusters are shown in Fig. 4 for samples of $\mathrm{Sm} / \mathrm{Ne}, \mathrm{Sm} / \mathrm{Ar}$, and $\mathrm{Sm} / \mathrm{Kr}$ at $T=5 \mathrm{~K}$. The valence curve as a function of $\log _{10}(M / R)$ displays a discontinuous transition from $v=2.6$ to $v=2.1$ at $M / R \sim 100: 1$. For higher metal concentrations 


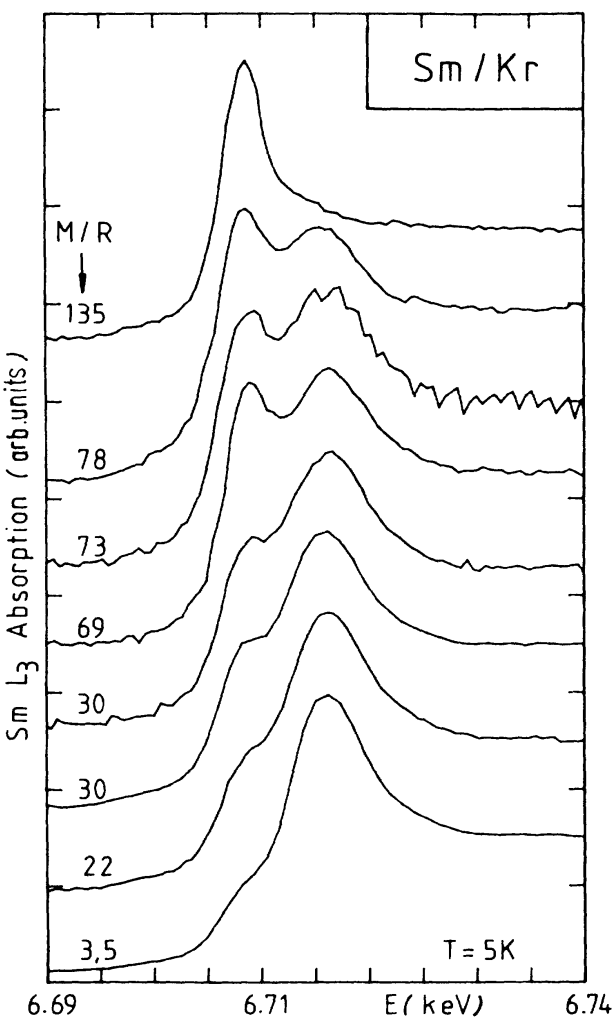

FIG. 3. $L_{3}$ absorption spectra of Sm clusters in krypton matrices at $T=5 \mathrm{~K}$. Thickness of Al support is $5 \mu \mathrm{m}$.

$(M / R<100)$ a linear increase of the valence with increasing metal concentration is observed. Except for $\mathrm{Ne}$, all samples with $M / R>400$ are completely divalent. The case of $\mathrm{Sm}$ in $\mathrm{Ne}$ needs some special attention. As $\mathrm{Ne}$ sublimes already at $8.8 \mathrm{~K}$, special care has to be taken for the sample preparation. Very low evaporation rates and a thick Al support are required. The valence still depends on the thickness of the Al foil. Because of different heat conductions, diffusion of the particles on the sample sur-

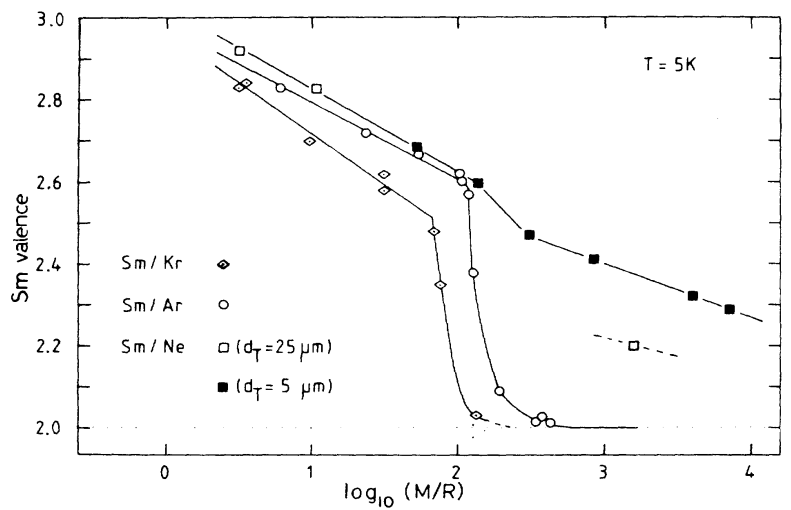

FIG. 4. Mean valence of $\mathrm{Sm}$ clusters isolated in matrices of $\mathrm{Ne}$, Ar, and $\mathrm{Kr}$. The $\mathrm{Sm}$ valence is shown as a function of the atomic concentration $M / R$ (matrix to radical). For the case of $\mathrm{Ne}$ matrices, different thicknesses of the Al support are given. face during the evaporation procedure leads to different mean cluster sizes. Within the thickness range $d_{T} \leq 25 \mu \mathrm{m}$ no completely divalent $\mathrm{Sm} / \mathrm{Ne}$ sample could be prepared for $M / R<2000$.

Annealing effects have been studied on all the $\mathrm{Sm} / \mathrm{Ne}$, $\mathrm{Sm} / \mathrm{Ar}$, and $\mathrm{Sm} / \mathrm{Kr}$ samples. It is well known that annealing of the samples may result in the consolidation of small aggregates to bigger ones or in the rearrangement of particles to structures which are energetically more suitable. If the annealing temperature is restricted to $T<T_{\text {crit }}$ where $T_{\text {crit }}$ is the temperature at which significant amounts of rare gas sublime, no important valence changes are observed ( $T_{\text {crit }}=32 \mathrm{~K}$ for Ar). The increase of valence $\Delta v$ is always limited to $\Delta v \leq 0.1$. Photoclustering effects ${ }^{24}$ by the $\mathrm{x}$-ray beam have not been observed.

EXAFS results: Determination of cluster size

EXAFS measurements in the transmission mode have been performed on the same samples which previously were used for XANES studies. Due mainly to the small

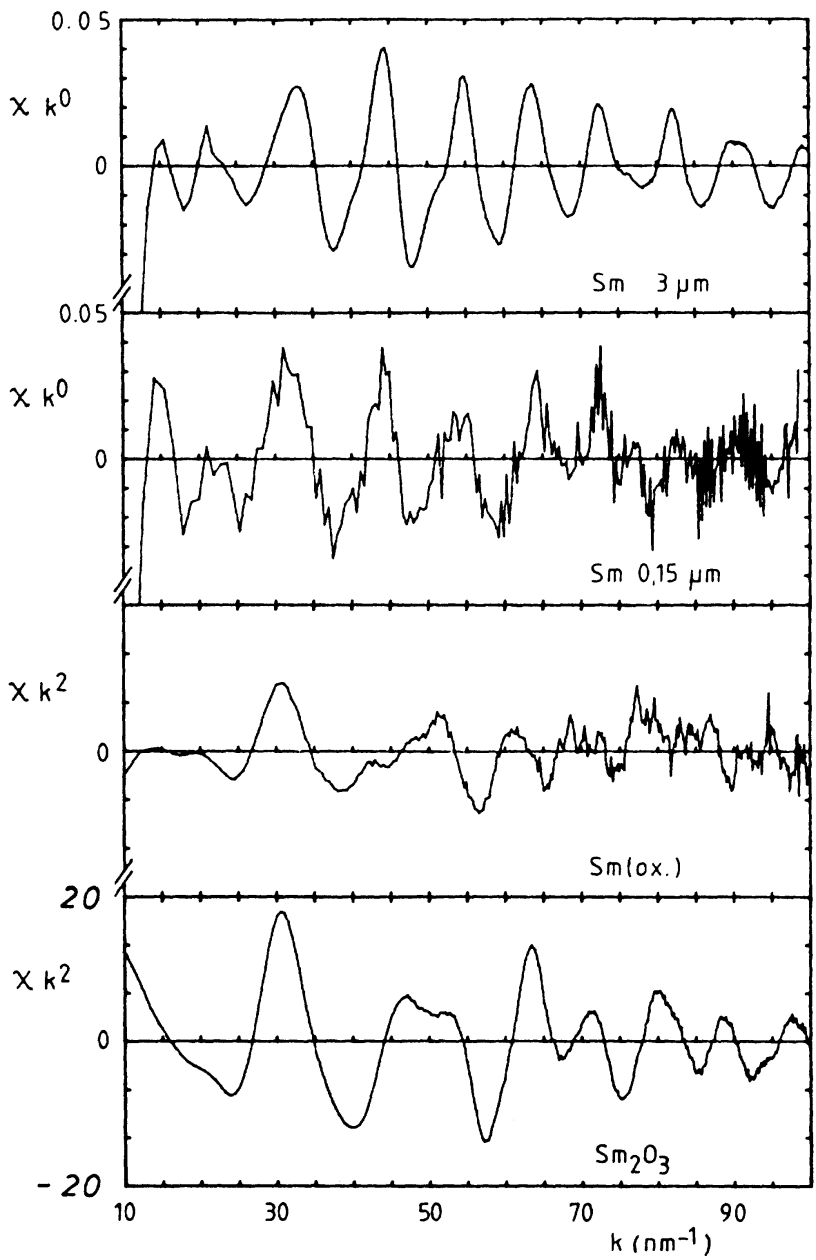

FIG. 5. $L_{3}$ absorption fine structures $\chi k^{0}$ of a Sm foil $(3 \mu \mathrm{m})$ and of an evaporated Sm layer $(0.15 \mu \mathrm{m})$, and fine structures $\chi k^{2}$ of a partially oxidized $\mathrm{Sm}$ sample and of $\mathrm{Sm}_{2} \mathrm{O}_{3}$. 
jump $\Delta \mu d \sim 0.05$ of the Sm $L_{3}$ edge being much less than the optimum value $\Delta \mu d \sim(1-2),{ }^{25}$ extreme difficulties were imposed on these measurements. Troubling effects of sample inhomogeneities due to nonuniform sample thickness have also been observed.

The structural information of the samples is contained in the EXAFS interference function $\chi(k)$, the normalized part of the $x$-ray-absorption coefficient [see Eq. (1)]. Being a function of the electron wave vector $k$, the equation for the extraction of the fine structure reads

$$
\chi(k)=\left[\mu(k)-\mu_{0}(k)\right] / \mu_{0}(k),
$$

with the smooth background $\mu_{0}(k)$.

From standard EXAFS theory we get $^{26,27}$

$$
\begin{gathered}
\chi(k)=(-1)^{l} \sum_{j} \frac{N_{j}}{k R_{j}^{2}} \exp \left(-2 \sigma_{j}^{2} k^{2}-2 R_{j} / \lambda_{j}\right) F_{j}(k) \\
\times \sin \left[2 k R_{j}+\Phi_{j}(k)\right] .
\end{gathered}
$$

$F_{j}(k)$ is the backscattering amplitude of each of the $N_{j}$ atoms of the $j$ th shell at distance $R_{j} . \sigma_{j}^{2}$ describes the

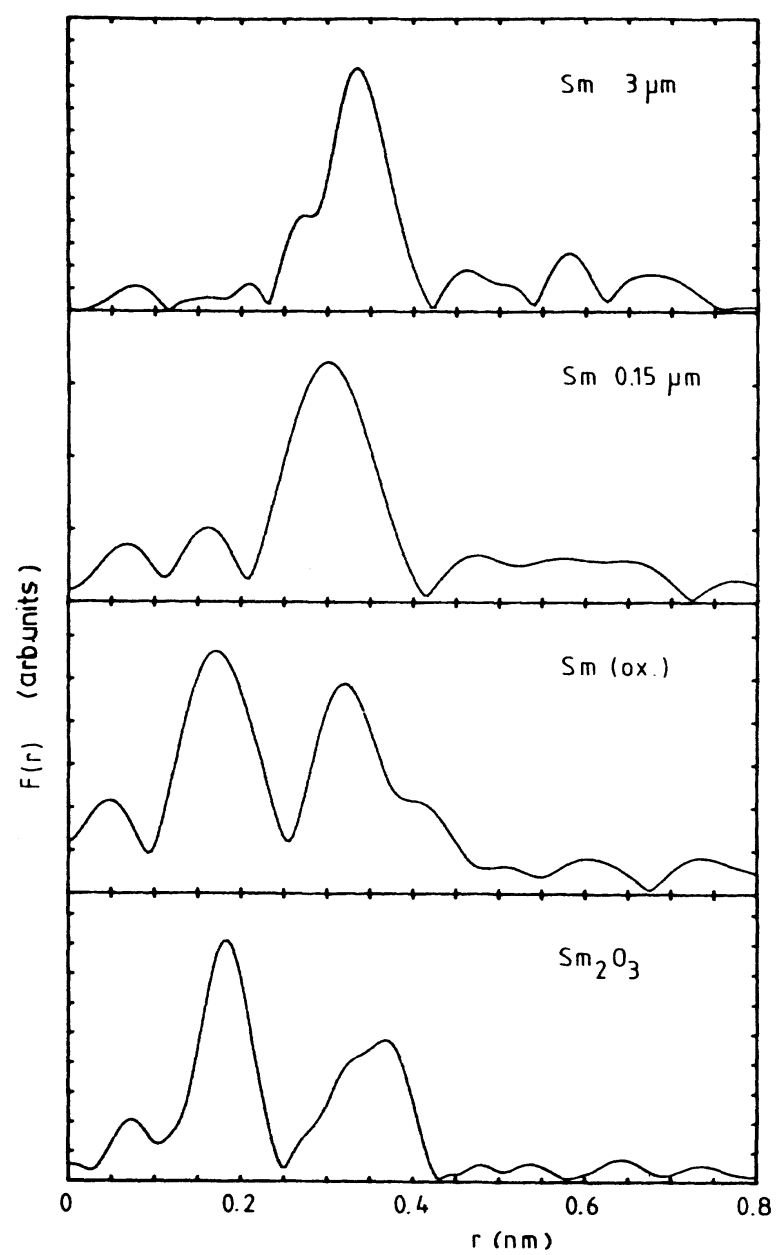

FIG 6. Fourier transforms of the fine structures shown in Fig. 5. mean-square displacement relative to the central absorbing atom. Inelastic processes are taken into account by the mean free path $\lambda_{j}$. The phase shift $\Phi_{j}(k)$ is due to the influence of the scattering potentials of the absorbing atom and the backscattering atom. Theoretical values for $F_{j}(k)$ and $\Phi_{j}(k)$ have been computed by Teo and Lee. ${ }^{28} 1$ is the angular momentum quantum number of the electron excited from its core level by the $x$-ray beam. The wave vector $k$ of the photoelectrons is determined by the dispersion relation

$$
k=\hbar^{-1}\left[2 m\left(E-E_{\mathrm{th}}\right)\right]^{1 / 2} .
$$

$E_{\text {th }}$ is the threshold energy.

By standard Fourier-transform techniques the structural information on one separate shell is obtained. First the normalized data are Fourier transformed into real space, and then the information belonging to one certain distance is obtained by back-transforming the corresponding peak in $F(r)$.

The parameters $N, R$, and $\sigma^{2}$ are determined by a linear least-squares fit to the back-transform data. For $\lambda$, the universal mean free path is taken in the same form as that described by Biebesheimer et al. ${ }^{29}$.

Before investigating the Sm clusters some measure-

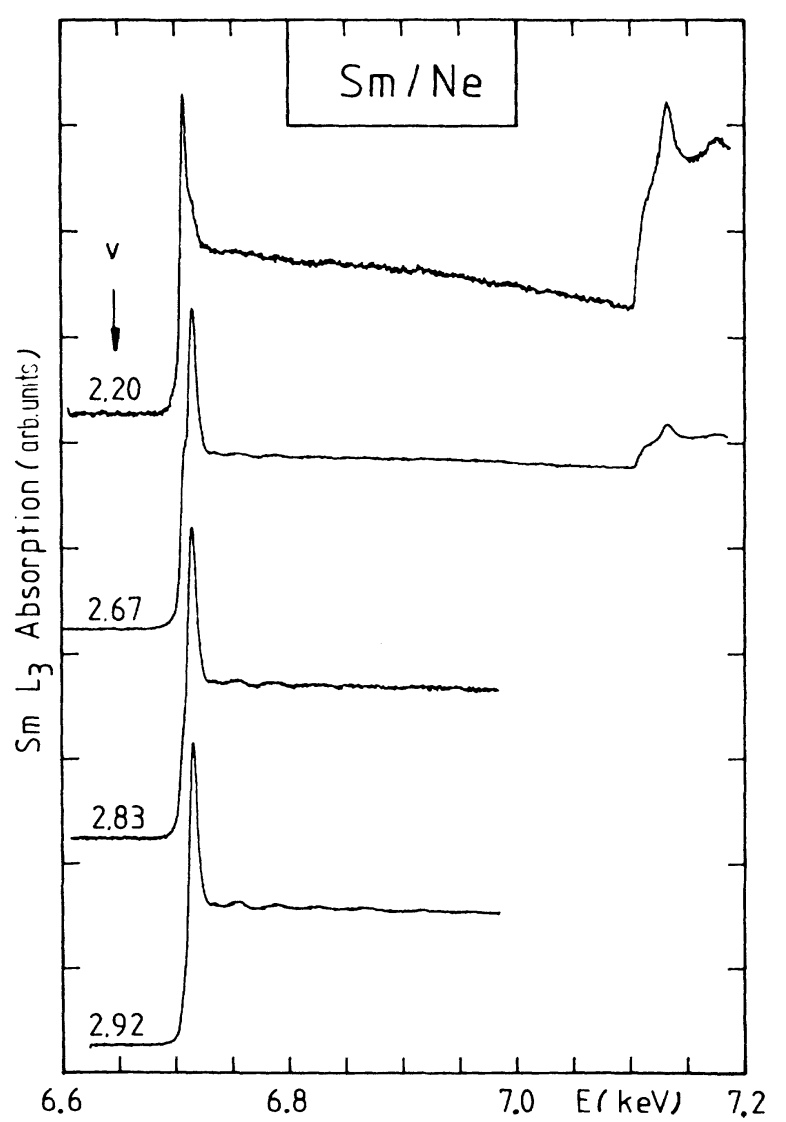

FIG. 7. $\mathrm{Sm} L_{3}$ absorption spectra for Sm clusters isolated in solid neon. The valences obtained by analysis of the near-edge structures are indicated. At $7.11 \mathrm{keV}$ the $K$ edge of iron impurities in the Al support appears. 
ments on reference data have been performed in order to test the quality of the sample preparation conditions and to get the experimental backscattering amplitude and phase shift $\Phi$ of Sm. Special attention has been drawn to the checking for impurities like oxygen or nitrogen which easily should be detected by EXAFS. Figure 5 shows the $L_{3}$ EXAFS $\chi k^{0}$ of a Sm foil with optimum thickness $(3 \mu \mathrm{m})$ and of a thin evaporated Sm film $(0.15 \mu \mathrm{m}$; $\Delta \mu d=0.035$ ) which has approximately the same metal thickness as the matrix samples. Additionally, the weighted fine structures $\chi k^{2}$ of a partially oxidized $\mathrm{Sm}$ film and of $\mathrm{Sm}_{2} \mathrm{O}_{3}$ are shown. In Fig. 6, the corresponding Fourier transforms are displayed.

The clean $\mathrm{Sm}$ sample shows only one peak around 0.3 $\mathrm{nm}$, whereas for oxidized samples an additional Sm-O peak around $0.18 \mathrm{~nm}$ appears. The existence of this peak can be used for the rejection of ill-conditioned matrix samples.

Using the well-known crystallographic data of crystalline $\mathrm{Sm},{ }^{30,31}$ the EXAFS of the $3-\mu \mathrm{m}$ Sm foil is used for the extraction of the experimental Sm backscattering amplitude and total phase $\Phi(k)$. In the region $k>100 \mathrm{~nm}^{-1}$, theoretical data ${ }^{28}$ have to be used because of the presence of the $\mathrm{Fe} K$ edge in the absorption data.

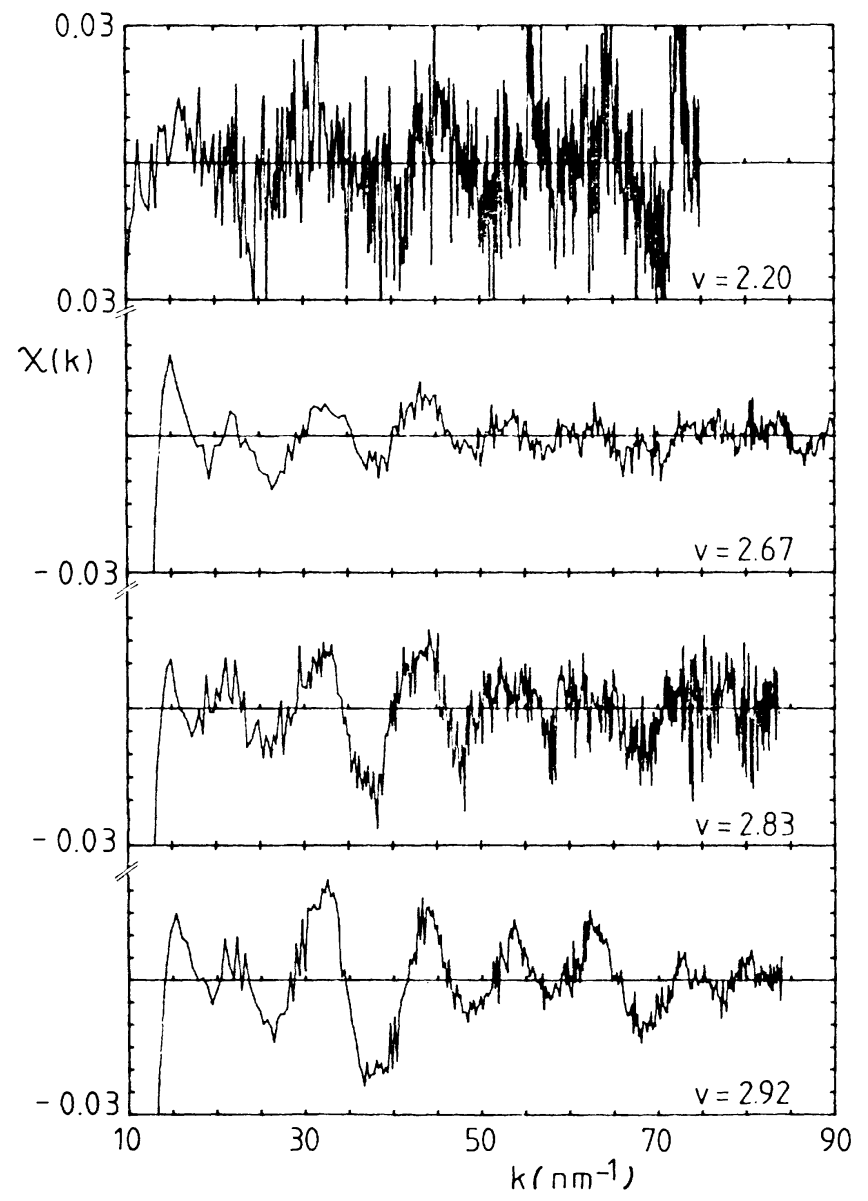

FIG. 8. $L_{3}$ EXAFS of $\mathrm{Sm}$ clusters isolated in solid $\mathrm{Ne}$ for mean valences $V=2.20,2.67,2.83$, and 2.92 .
In order to get the backscattering amplitude of $\mathrm{Sm}$, $\sigma^{2}=55 \mathrm{pm}^{2}$ has been used. The mean free path has been set to

$$
\lambda_{\text {uni }}=1.3+0.864 k+80.70 k^{-3.44},
$$

in order to guarantee the nearest-neighbor number of 12 atoms.

In spite of the bad signal-to-noise ratio of the thin sample, the fits of the 3-and $0.15-\mu \mathrm{m} \mathrm{Sm}$ samples yield nearly identical results. For the $0.15-\mu \mathrm{m}$ sample, $\sigma^{2}$ increases to $90 \mathrm{pm}^{2}$.

The absorption spectra of $\mathrm{Sm} / \mathrm{Ne}$ for valences $v=2.20$, 2.67, 2.83, and 2.92 are shown in Fig. 7. The range of information is limited to $E<7.1 \mathrm{keV}$ because of the presence of the $\mathrm{Fe} K$ edge which is due to $\mathrm{Fe}$ impurities in the Al support. The normalized fine structures of these spectra obtained after subtracting the smooth background $\mu_{0}$ are exposed in Fig. 8, where the decrease of the amplitude with decreasing $\mathrm{Sm}$ valence is clearly observed. The EXAFS for the $v=2.20$ sample is shown to demonstrate the difficulties entering the measurements for low metal concentrations. The fine structures of this sample are not EXAFS but due to sample inhomogeneities. Sample inhomogeneities cause additional fine structures because of small movements of the $\mathrm{x}$-ray focus on the sample. The tendency of decreasing amplitude is also clearly demonstrated by inspecting the corresponding Fourier transforms in Fig. 9. A large Sm-Sm peak is observed at

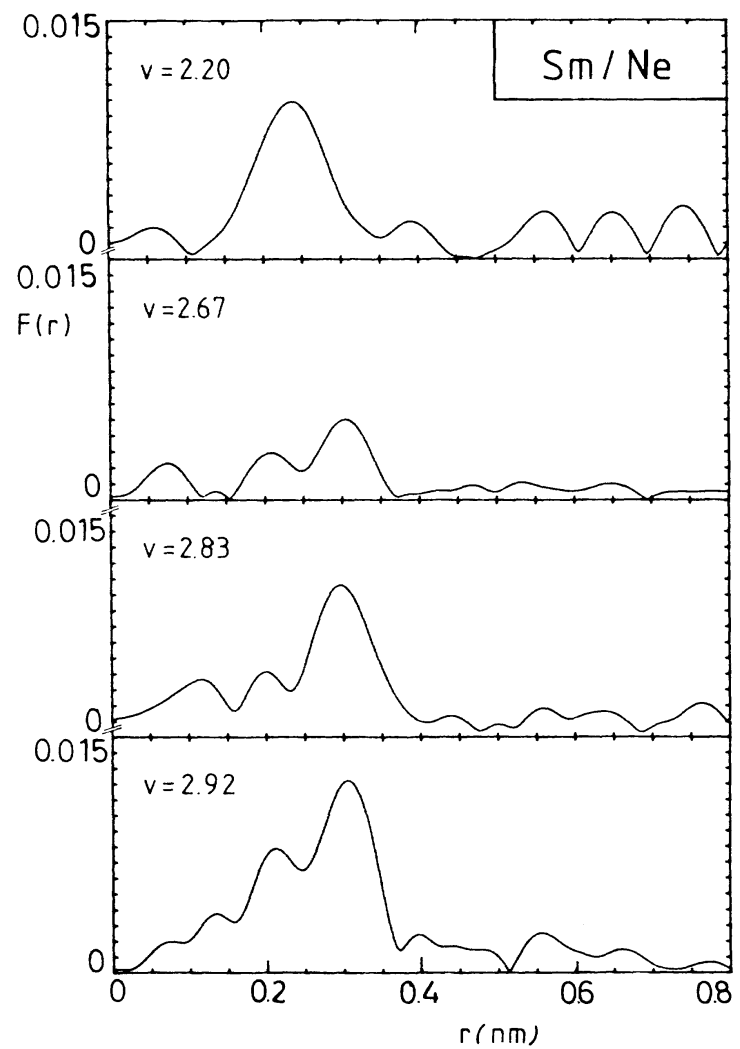

FIG. 9. Fourier transforms of the $\mathrm{Sm} / \mathrm{Ne}$ fine structures shown in Fig. 8. 
$r=0.31 \mathrm{~nm}$. A second peak shows up around $r=0.21$ $\mathrm{nm}$. A close inspection of the information contained in this peak shows that it is not due to $\mathrm{Sm}-\mathrm{O}$ distances. The envelope of the corresponding EXAFS is a nearly constant function so that impurities with low $Z$ can be neglected. A critical analysis shows that this peak is due to certain irregular movements of the monochromator which could also be detected in spectra taken without a matrix on the sample holder. ${ }^{15}$

The EXAFS results of the $\mathrm{Sm} / \mathrm{Ar}$ and $\mathrm{Sm} / \mathrm{Kr}$ systems are very similar to those of the $\mathrm{Sm} / \mathrm{Ne}$ system. We have succeeded in doing EXAFS measurements for samples with $v>2.5$. For lower valences all the attempts of measuring reliable EXAFS failed because of the low coordination and because of a bad signal-to-noise ratio. It turns out that the EXAFS of the matrix samples is independent of the matrix material for constant valence.

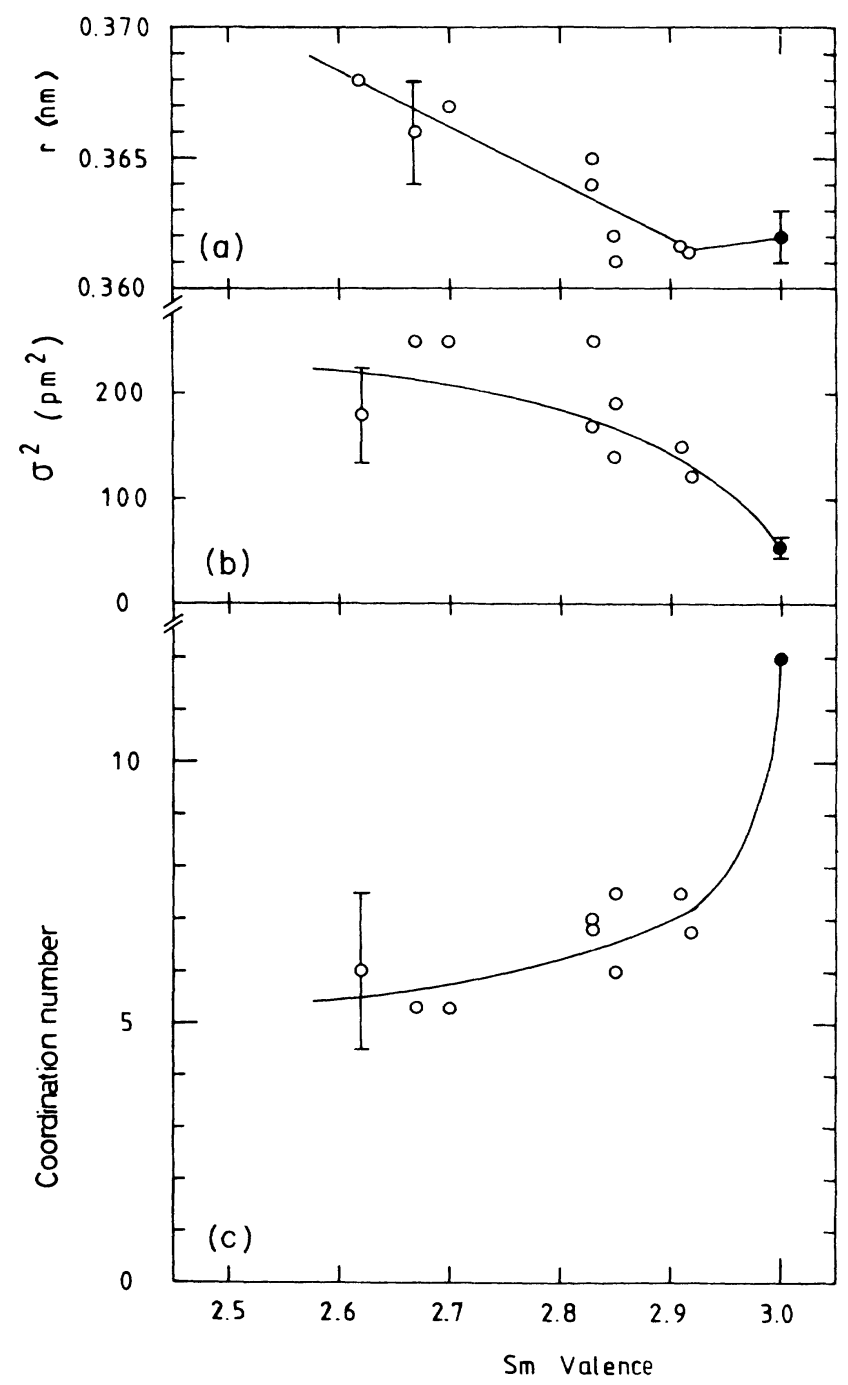

FIG. 10. EXAFS fit results for Sm clusters isolated in matrices of solid rare gases $\mathrm{Ne}, \mathrm{Ar}$, and $\mathrm{Kr}$. The nearest-neighbor distance $r$, the mean-square displacement $\sigma^{2}$, and the coordination number $N$ are drawn as a function of the mean $\mathrm{Sm}$ valence. Values for solid Sm with $v=3.0$ are shown by solid circles $(\bullet)$.
No Sm-rare-gas pairs could be detected, only Sm-Sm pairs. Using the experimental $\mathrm{Sm}$ backscattering amplitude and the experimental Sm total phase, the backtransform of the $0.31-\mathrm{nm}$ peak has been fitted by a oneshell Sm-Sm fit. According to common practice $32-34$ only one threshold energy is used for these mixed-valent systems. The results of the fit procedure are shown in Fig. 10. The nearest-neighbor distance $r$, the mean-square displacement $\sigma^{2}$, and the coordination number $N$ are displayed as a function of the mean cluster valence $v$. Data for all matrices of $\mathrm{Ne}, \mathrm{Ar}$, and $\mathrm{Kr}$ are included in this figure. The threshold energy had to be varied within a range of typically $\pm 1 \mathrm{eV}$.

A linear increase of nearest-neighbor distance with decreasing valence (equal to decreasing cluster size) is observed. The static disorder of the samples also increases with decreasing valence. The coordination number $N$ drops down from $N=12$ for the solid to $N=5-6$ for clusters with $v=2.6$. Assuming a fcc structure for small Sm clusters, a mean cluster size $S=13 \pm 3$ atoms follows.

The static disorder of the samples may be explained by different geometrical cluster configurations and by incomplete crystallization due to the condensation at very low temperatures $(5 \mathrm{~K})$. A decrease of the nearest-neighbor distance with decreasing cluster size due to surface stress effects $^{35}$ has been observed by EXAFS measurements on some metal clusters (see, e.g., Refs. 36-38). For the case of $\mathrm{Sm}$, the reverse tendency is stated in this paper. The reason for that is the bigger atomic radius of divalent $\mathrm{Sm} .^{12}$

\section{DISCUSSION: SIZE-DEPENDENT VALENCE OF MATRIX-ISOLATED Sm CLUSTERS}

From the combination of EXAFS and XANES results the size-dependent valence of $\mathrm{Sm}$ clusters isolated in matrices of $\mathrm{Ne}$, Ar, and $\mathrm{Kr}$ can be discussed. Using the well-known valences 2 (3) of ${ }^{4-6}$ surface (bulk) samarium it is easy to show that a cluster size $S=1000$ atoms is needed for a mean valence $v=2.5$. Photoemission studies on evaporated $\mathrm{Sm}$ layers ${ }^{7}$ have revealed a transition from mainly divalent to mainly trivalent samples at a coverage of $(3-4) \times 10^{15}$ atoms $/ \mathrm{cm}^{2}$. The corresponding cluster size was determined by electron microscopy to be $\sim 3 \mathrm{~nm}$. This cluster size is in agreement with the estimated value $S_{c}=1000$ atoms. The critical cluster size $S_{c}=13$ atoms of the matrix-isolated $\mathrm{Sm}$ clusters is in contrast to the formerly mentioned value.

As the valence transition is very sharp, we conclude that $\mathrm{Sm}$ clusters with $S<10$ atoms are totally divalent. For $S \geq 13$, a linear increase of valence with decreasing $M / R$ is observed, the cluster valence approaching the Sm bulk valence for low $M / R$ values. The critical cluster size $S_{c}=13$ corresponds to the first "magic number" observed in free xenon cluster beams by Echt et al. ${ }^{39}$ The cluster size 13 is distinguished by a closed shell of 12 atoms surrounding a single central atom. This geometrical structure results in a particular stability.

From the surprisingly low critical cluster size $S_{c}=13$ for matrix-isolated Sm clusters, a mixed valence of the atoms follows for clusters with $S_{c} \geq 13$. This can only be 


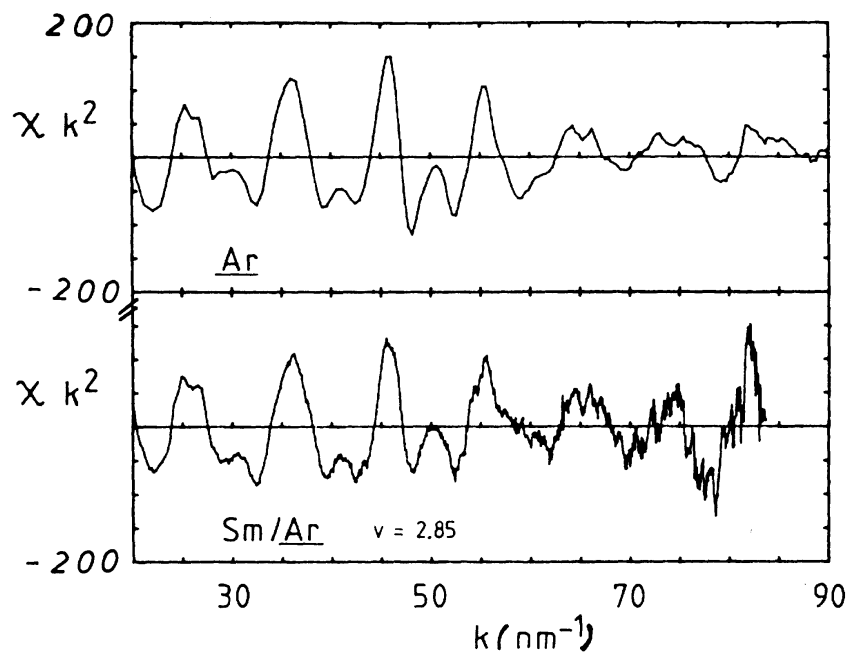

FIG. 11. Weighted fine structures $\chi k^{2}$ of solid $\mathrm{Ar}$ and of $\mathrm{Sm} / \operatorname{Ar}(v=2.85)$ at the $\operatorname{Ar} K$ edge for $T=5 \mathrm{~K}$.

explained by the influence of the matrix. This influence is demonstrated in two effects. The first effect is an observable connection between the valence $v_{c}$ at the transition and the kind of rare gas used as a matrix material. It can easily be seen from Fig. 4 that the critical valence $v_{c}$ takes values 2.60 or 2.55 for matrices of neon, argon, or krypton. The second effect is the contraction of the rare-gas lattice due to the embedding of the clusters. This contraction has been determined by EXAFS measurements at the Ar $K$ edge for samples of $\mathrm{Sm} / \mathrm{Ar}$.

In Fig. 11 the weighted fine structures $\chi k^{2}$ of solid Ar and of $\operatorname{Sm} / \operatorname{Ar}(v=2.85)$ are shown. The corresponding

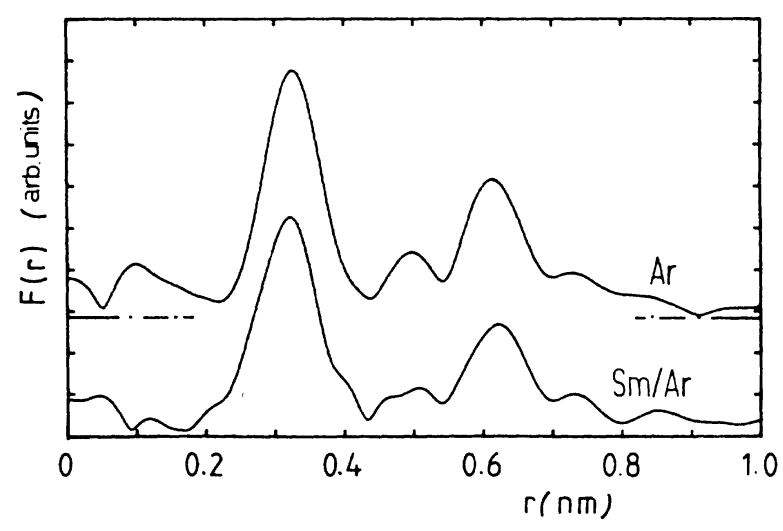

FIG. 12. Fourier transforms of the fine structures shown in Fig. 11.

Fourier transforms exposed in Fig. 12 are nearly identical. The first peak near $r=0.33 \mathrm{~nm}$ is attributed to the nearest Ar-Ar distance. For the matrix, no Ar-Sm contribution could be detected. From a fit to the first shell, a mean nearest-neighbor distance $R_{\mathrm{Ar}-\mathrm{Ar}}=0.375 \mathrm{~nm}$ is determined for the pure Ar sample, whereas in the matrix we find $R_{\mathrm{Ar}-\mathrm{Ar}}=0.370 \mathrm{~nm}$. This lattice contraction must be due to the $\mathrm{Sm}$ clusters. Similar effects have been observed for matrix-isolated rare-gas clusters. ${ }^{14}$

\section{ACKNOWLEDGMENTS}

This work has been supported by the Bundesministerium für Forschung und Technologie (BMFT) under Contract No. 05-245-RA. The authors would like to thank B. Sonntag for helpful discussions.
${ }^{*}$ Present address: Haldor Topsøe Research Laboratories, DK2800 Lyngby, Denmark.

†resent address: IBM Thomas J. Watson Research Center, P.O. Box 218, Yorktown Heights, NY 10598.

$\ddagger$ Also at Fachhochschue Ostfriesland, D-2970 Emden, Federal Republic of Germany.

§Present address: Institut Laue-Langevin, 38042 Grenoble, France.

${ }^{* *}$ Present address: C. H. F. Müller, Unternehmensbereich der Philips G.m.b.H., D-2000 Hamburg 63, Federal Republic of Germany.

${ }^{1}$ Valence Fluctuations in Solids, edited by L. M. Falicov, W. Hanke, and M. P. Maple (North-Holland, Amsterdam, 1981).

${ }^{2}$ Valence Instabilities, edited by $\mathrm{P}$. Wachter and $\mathrm{H}$. Boppart (North-Holland, Amsterdam, 1982).

${ }^{3}$ Proceedings of the 4th International Conference on Valence Fluctuations, edited by E. Müller-Hartmann, B. Roden, and D. Wohleben [J. Magn. Magn. Mater. $47 \& 48$ (1985)].

${ }^{4}$ G. K. Wertheim and G. Crecelius, Phys. Rev. Lett. 40, 813 (1978).

5J. K. Lang and Y. Baer, Solid State Commun. 31, 945 (1979).

${ }^{6} \mathrm{~F}$. Gerken, A. S. Flodström, L. I. Johansson, and C. Kunz, Physica Scr. 32, 43 (1985).
${ }^{7}$ M. G. Mason, S. T. Lee, G. Apai, R. F. Davis, D. A. Shirley, A. Franciosi, and J. H. Weaver, Phys. Rev. Lett. 47, 730 (1981).

${ }^{8}$ A. Fäldt and H. P. Myers, J. Magn. Magn. Mater. 47\& 48, 225 (1985).

${ }^{9}$ M. Domke, C. Laubschat, M. Prietsch, T. Mandel, G. Kaindl, and W. D. Schneider, Phys. Rev. Lett. 12, 1287 (1986).

${ }^{10}$ A. Rosengren and B. Johansson, Phys. Rev. B 26, 3068 (1982).

${ }^{11}$ W. Niemann, M. Lübcke, W. Malzfeldt, P. Rabe, and R. Haensel, J. Magn. Magn. Mater. 47\& 48, 462 (1985).

${ }^{12}$ M. Lübcke, B. Sonntag, W. Niemann, and P. Rabe, Phys. Rev. B 34, 5184 (1986).

${ }^{13}$ W. Malzfeldt, W. Niemann, R. Haensel, and P. Rabe, Nucl. Instrum. Methods 208, 359 (1983).

${ }^{14}$ W. Malzfeldt, Ph.d dissertation, Universität Kiel, 1985.

15W. Niemann, Ph.d dissertation, Universität Kiel, 1985.

16J. Cerino, J. Stöhr, N. Hower, and R. Z. Bachrach, Nucl. Instrum. Methods 172, 227 (1980).

${ }^{17}$ R. E. Honig, RCA Rev. 23, 567 (1962).

${ }^{18}$ W. J. Veigele, in Handbook of Spectroscopy (Chemical Rubber Co., Cleveland, 1974), Vol. 1.

19J. A. Victoreen, J. Appl. Phys. 19, 855 (1948).

${ }^{20}$ E. E. Vainshtain, S. M. Blokhin, and Yu. B. Paderna, Fiz. 
Tverd. Tela (Leningrad) 6, 2909 (1964). [Sov. Phys.-Solid State 6, 2318 (1965)].

${ }^{21}$ G. Materlik, B. Sonntag, and M. Tausch, Phys. Rev. Lett. 51, 1300 (1983).

22J. F. Kielkopf, J. Opt. Soc. Am. 63, 987 (1973).

23J. Röhler, J. Magn. Magn. Mater. 47\& 48, 175 (1985).

${ }^{24}$ G. A. Ozin and H. Huber, Inorg. Chem. 17, 155 (1978).

${ }^{25}$ P. A. Lee, P. H. Citrin, P. Eisenberger, and B. M. Kincaid, Rev. Mod. Phys. 53, 769 (1981).

${ }^{26}$ E. A. Stern, Phys. Rev. B 10, 3027 (1974).

${ }^{27}$ E. A. Stern, D. E. Sayers, and F. W. Lytle, Phys. Rev. B 11, 4836 (1975).

${ }^{28}$ B.-K. Teo and P. A. Lee, J. Am. Chem. Soc. 101, 2815 (1979).

${ }^{29}$ V. A. Biebesheimer, E. C. Marques, D. R. Sandstrom, F. W. Lytle, and R. B. Greegor, J. Chem. Phys. 81, 2599 (1984).

${ }^{30}$ A. H. Daane, R. E. Rundle, H. G. Smith, and F. H. Spedding, Acta Crystallogr. 7, 532 (1954).

${ }^{31}$ A. J. Beaudry and P. E. Palmer, J. Less-Common Met. 34, 225 (1974).

32J. B. Boyce, R. M. Martin, and J. W. Allen, in EXAFS and
Near Edge Structure, edited by A. Bianconi, L. Incoccia, and S. Stipcick (Springer, Berlin, 1983), p. 187.

${ }^{33}$ K. H. Frank, G. Kaindl, J. Feldhaus, G. Wortmann, W. Krone, G. Materlik, and $\mathrm{H}$. Bach, in Valence Instabilities, edited by P. Wachter and H. Boppart (North-Holland, Amsterdam, 1982). p. 189.

${ }^{34}$ G. Krill, J. P. Kappler, M. F. Ravet, J. Röhler, C. Godart, and J. P. Senateur, J. Magn. Magn. Mater. 47\& 48, 190 (1985).

35J. S. Vermaak, C. W. Mays, and D. Kuhlmann-Wilsdorf, Surf. Sci. 12, 128 (1968).

36P. A. Montano, W. Schulze, B. Tesche, G. K. Shenoy, and T. I. Morrison, Phys. Rev. B 30, 672 (1984).

${ }^{37}$ G. Apai, J. F. Hamilton, J. Stöhr, and A. Thompson, Phys. Rev. Lett. 43, 165 (1979).

${ }^{38}$ A. Balerna, E. Bernieri, P. Picozzi, A. Reale, S. Santucci, E. Burattini, and S. Mobilio, Phys. Rev. B 31, 5058 (1985).

${ }^{39}$ O. Echt, K. Sattler, and E. Recknagel, Phys. Rev. Lett. 47, 1121 (1981). 